\title{
Visceral Adiposity Index and Atherogenic Index of Plasma as Useful Predictor of Cardiovascular Diseases Risk: Evidence From A Cohort Study in Iran
}

\section{Behrooz Hamzeh}

Kermanshah University of Medical Sciences

Yahya Pasdar

Kermanshah University of Medical Sciences

Narmin Mirzaei

Kermanshah University of Medical Sciences

Roya Safari Faramani

Kermanshah University of Medical Sciences

Farid Najafi

Kermanshah University of Medical Sciences

Ebrahim Shakiba

Kermanshah University of Medical Sciences

Mitra Darbandi ( $\nabla$ m.darbandi@kums.ac.ir)

Kermanshah University of Medical Sciences

\section{Research Article}

Keywords: Cardiovascular diseases, Body Mass Index, Primary Prevention, Cholesterol, Obesity

Posted Date: June 15th, 2021

DOI: https://doi.org/10.21203/rs.3.rs-609029/v1

License: (9) This work is licensed under a Creative Commons Attribution 4.0 International License.

Read Full License

Version of Record: A version of this preprint was published at Lipids in Health and Disease on August 1st, 2021. See the published version at https://doi.org/10.1186/s12944-021-01505-w. 


\section{Abstract \\ Background}

Visceral Adiposity index (VAI) and atherogenic index of plasma (AIP) are relatively new indicators for predicting Non-Communicable disease (NCDs). The aim of this study was to assess the association AIP and VAI with risk of cardiovascular diseases (CVDs).

\section{Methods}

This was a cross-sectional analysis conducted on 7362 individuals aged 35 to 65 years participated in Ravansar Non-Communicable Diseases (RaNCD) cohort study. AIP was calculated based on the value of triglyceride and high density lipoprotein cholesterol (HDL-C). VAI was calculated using Body mass index (BMI), waist circumference (WC), triglyceride, and HDL-C. All participants were stratified into three groups based on AIP and VAI tertiles. Logistic regression models were used to assess the association of AIP and VAl with CVDs.

\section{Results}

The mean of AIP and VAI was significantly higher in CVDs patients than in non-CVDs $(P<0.001)$. After adjusting for age, sex, BMI and physical activity the risk of CVDs in the second and third tertile of AIP were $1.22(95 \% \mathrm{Cl}: 1.02,1.45)$ and $1.40(95 \% \mathrm{Cl}: 1.19,1.66)$ times higher comparing to the first tertile, respectively. Risk of CVDs in the second and third tertile of VAl were $1.28(95 \% \mathrm{Cl}: 1.06,1.53)$ and 1.52 $(95 \% \mathrm{Cl}: 1.25,1.83)$ times higher than the first tertile, respectively; while adjusting for age, sex, hypertension and dyslipidemia and smoking.

\section{Conclusion}

According to the findings, AIP and VAI were positively associated with CVDs. Therefore, AIP and VAI can be useful in identifying high-risk subgroups of CVDs in general population.

\section{Background}

Cardiovascular diseases (CVDs) are the leading cause of mortality worldwide. According to the global burden of diseases (GBD), CVDs are the cause of 17.9 million deaths in 2017, of which more than threequarters were in low- and middle-income countries [1]. Approximately $50 \%$ of annual deaths and $50 \%$ of deaths caused by non-communicable diseases (NCDs) are due to CVDs in Iran [2].

In addition to genetic factors, there are several known risk factors for CVDs event, including hypertension; dyslipidemia, obesity, and smoking, some of these factors are almost preventable and easy to change [3]. 
Reducing blood pressure and serum cholesterol can, indeed, effectively prevent or delay CVDs events [4]. Thus, measuring the risk factors for prediction and early detection of disease can be useful. In a metaanalysis study done in 2020, CVDs prediction was reported based on simple anthropometric indices including waist circumference (WC), body mass index (BMI) and waist to hip ratio (WHR) [5]. Accumulation of visceral fat and blood lipid also are among the numerous risk factors contributing to CVDs $[6,7]$.

Amato et al. ntroduced visceral adiposity index (VAl), which is a combination of WC, BMI, triglyceride (TG) and high-density lipoprotein cholesterol (HDL-C) [8]. VAI is a reliable index for the function of visceral fat in the body. Previous studies have confirmed the accuracy of VAl for predicting NCDs including metabolic syndrome (MetS), type 2 diabetes mellitus (T2DM) and hypertension [9-11].

Among the biochemical indicators that represent the status of blood lipids, the atherogenic index of plasma (AIP) is the logarithmic conversion of the TG to HDL-C ratio, a strong predictor of atherosclerosis and CVDs. In addition, AIP correlates with lipoprotein particle size including high-density lipoprotein cholesterol (LDL-C), HDL-C and very-low-density lipoprotein (VLDL) which act as the most sensitive CVDs markers; Moreover, it as a reliable and accurate predictor of heart attack and atherosclerosis [12-14]. AIP is the best determinant for fractionated esterification rate of HDL-C and more useful than routine lipid markers. Previous research has shown that the average of these two indices is different in different populations; the mean of AIP is between 0.17 to $0.41[13,15]$ and the mean of VAl is between 2.1 to 3.2 in Iranian populations [16-18]. The difference in the averages of these indexes indicates the need for further studies in different populations and ethnicities. Thus, using accurate and low-cost indicators is useful to evaluate the risk factors of CVDs and its prediction. Therefore, this study aimed to assess the association of AIP and VAI with CVDs in adults using the Iranian Ravansar Non-Communicable Diseases (RaNCD) Cohort Data.

\section{Methods}

\section{Study design and participants}

This cross-sectional study was conducted using data from Ravansar non-communicable diseases (RaNCD) cohort study. The RaNCD cohort study is a part of the Prospective Epidemiological Research Studies of Iranian Adult (PERSIAN) cohort. In the PERSIAN cohort, all 19 cohort sites (covering a representative sample of different Iranian ethnicities) used the same questionnaire and aimed to follow up all participants for the next 15 years. Further information is available at http://persiancohort.com. Ravansar is a district with a population of about 50,000 people, located in western Iran and in Kermanshah province. The number of participants in the baseline phase of the RaNCD was 10,000 adults, who all of whom were permanent residents of Ravansar. Details of the RaNCD methodology has been described elsewhere [19].

\section{Inclusion and exclusion criteria}


All subjects in the initial phase of RaNCD entered the present study. According to the objectives of the present study, subjects with cancer $(n=85)$, renal failure $(n=64)$, kidney stones $(n=1794)$, pregnant woman $(n=138)$ and cases with missed information $(n=557)$ were excluded from the study, finally 7362 subjects remained (Figure 1).

\section{Data collection}

All interviews were conducted by trained and face-to-face individuals at the RaNCD cohort Study Center. Demographic information including age, sex, smoking and history of chronic diseases were recorded online in an electronic data collection form. Biochemical parameters, anthropometric indices and blood pressure were measured according to the PERSIAN cohort protocol.

\section{Physical activity}

Physical activity measured using metabolic equivalent rates (METs) of self-reported daily activities of participants of PERSIAN cohort using the questionnaire, including 22 questions about their sport, work, and leisure- related activities on an average weekday. The questionnaire information was extracted and used based on Met/ hour per week. [20].

\section{Blood pressure measurements}

Blood pressure was measured using a manual sphygmomanometer (Riester) from both arms, sitting position and after 10 minutes of rest, and its mean was reported. Hypertension was defined as subjects with systolic blood pressure (SBP) $\geq 140$ and diastolic blood pressure (DBP) $\geq 90$ or current use of medication for hypertension [21].

\section{Anthropometric measurements}

Body weight was measured using Bio-Impedance Analyzer (BIA) model of (Inbody 770, Inbody Co, Seoul, Korea) with a precision of $0.5 \mathrm{~kg}$. Other anthropometric measurements including body fat mass (BFM), percent body fat (PBF), skeletal muscle mass (SLM) and visceral fat area (VFA) were also measured with BIA. The height of the participants was measured with BSM 370 (Biospace Co, Seoul, Korea) with a precision of $0.1 \mathrm{~cm}$. $B M /$ was calculated weight $(\mathrm{kg})$ divided by the square of height $(\mathrm{m})$. WC was measured with a flexible measuring tape at a level midway between the lower rib margin and the iliac crest to the nearest $0.5 \mathrm{~cm}$. VAl was measured by the following formula [8]:

$$
\begin{aligned}
& \text { Males: VAI }=\left(\frac{W C}{39.68+(1.88 \times B M I)}\right) \times\left(\frac{T G}{1.03}\right) \times\left(\frac{1.31}{H D L}\right) \\
& \text { Females: VAI }=\left(\frac{W C}{39.58+(1.89 \times B M I)}\right) \times\left(\frac{T G}{0.81}\right) \times\left(\frac{1.52}{H D L}\right)
\end{aligned}
$$

\section{Biochemical measurements}


The blood samples were collected after 8- 12 hours fasting, to measure biochemical markers including lipid profile (TG, LDL-C, HDL-C, Total cholesterol) and fasting blood sugar (FBS) and Liver enzymes including Alkaline phosphatase (ALP), Aspartate transaminase (AST), Alanine aminotransferase (ALT), Gamma-glutamyl transferase (GGT). AIP was calculated by using the following formula: $\log _{10}$ (TG/HDLC) [14]; and can be classified based on the values obtained: -0.3 to 0.1 for low risk, 0.1 to 0.24 for moderate, and $0.24<$ for high risk of CVDs [22]. Dyslipidemia was defined as LDL-C $\geq 160 \mathrm{mg} / \mathrm{dL}$ and/or total cholesterol $\geq 240 \mathrm{mg} / \mathrm{dl}$ and/ or HDL- $\mathrm{C}<40 \mathrm{mg} / \mathrm{dl}$ and/or TG $\geq 200 \mathrm{mg} / \mathrm{dl}$ and/or having a history of medication for this condition [21]. The MetS was defined according to International Diabetes Federation (IDF) criteria [23].

\section{Definition of outcome}

Participants who had at least one of the following condition were considered as patients with CVDs: a history of ischemic heart disease (IHD), heart failure and angina, stroke, Myocardial Infarction (MI) and/or current use of medication for CVDs.

\section{Data analysis}

Data analysis was performed using Stata version 14.1 software (Stata Corp, College Station, TX, USA). In descriptive reports, quantitative variables are presented as mean \pm standard deviation and qualitative variables as frequency (percentage). We compared baseline characteristics of studied participants by Chisquare and T-test between CVDs and non-CVDs groups. We used one-way ANOVA test to compare the variables in AIP and VAI tertiles. According to the binary outcome (CVDs), we used simple and multiple logistic regression models to assess the association between AIP and VAI with CVD, variables with $p$ value $<0.2$ in univariable analysis were entered into multivariable logistic model. The crude and adjusted odds ratios with $95 \%$ confidence interval were reported. The $P$ - value $<0.05$ was considered to be statistically significant in all statistical tests.

\section{Results}

Finally, 7,362 subjects out of 10,000 participants of RaNCD were eligible for data analysis. The baseline demographics, biochemical and anthropometrics characteristics of the participants are shown in Table 1. The mean age of the participants was $47.20 \pm 8.33$ years and $3420(46.45 \%)$ were male. The mean of VAI for participants with CVDs was significantly higher compared to participants without CVDs, $(P<0.001)$. The mean AIP in CVDs and non-CVDs groups were $1.07 \pm 0.61$ and $0.94 \pm 0.65$, respectively $(P<0.001)$. Other anthropometric indices, TG, LDL-C, total cholesterol, FBS, ALP and GGT were significantly higher in CVDs patients compared to non-CVDs groups. The prevalence of MetS in the CVDs group (53.80\%) was significantly higher than the non-CVDs group $(20.37 \%),(P<0.001)$. 
Table 1

Baseline characteristics according to cardiovascular disease (CVDs) status

\begin{tabular}{|c|c|c|c|c|}
\hline Parameters & $\begin{array}{l}\text { Total } \\
(n=7362)\end{array}$ & $\begin{array}{l}\text { Non-CVDs } \\
(n=6191)\end{array}$ & $\begin{array}{l}\text { CVDs } \\
(n=1171)\end{array}$ & $P$ value \\
\hline \multicolumn{5}{|l|}{ Gender, n (\%) } \\
\hline Male & $3420(46.45)$ & 3036 (49.04) & $384(32.79)$ & \multirow[t]{2}{*}{$<0.001$} \\
\hline Female & 3942 (35.55) & $3155(50.96)$ & $787(67.21)$ & \\
\hline Age (year) & $47.20 \pm 8.33$ & $46.03 \pm 7.90$ & $53.39 \pm 7.79$ & $<0.001$ \\
\hline Current smoker, n (\%) & $865(22.33)$ & $764(23.45)$ & $101(16.40)$ & $<0.001$ \\
\hline \multicolumn{5}{|l|}{ Physical activity, n (\%) } \\
\hline Low & $2168(29.45)$ & $1736(28.04)$ & $432(36.89)$ & \multirow[t]{3}{*}{$<0.001$} \\
\hline Moderate & 3539 (48.07) & 2979 (48.12) & $560(47.82)$ & \\
\hline Vigorous & $1655(22.48)$ & $1476(23.84)$ & 179 (15.29) & \\
\hline BMI $\left(\mathrm{kg} / \mathrm{m}^{2}\right)$ & $27.44 \pm 4.67$ & $27.20 \pm 4.63$ & $28.69 \pm 4.66$ & $<0.001$ \\
\hline WHR & $0.94 \pm 0.06$ & $0.93 \pm 0.06$ & $0.95 \pm 0.06$ & $<0.001$ \\
\hline WC (cm) & $96.92 \pm 10.55$ & $96.28 \pm 10.43$ & $100.30 \pm 10.59$ & $<0.001$ \\
\hline BFM (kg) & $24.99 \pm 9.62$ & $24.42 \pm 9.54$ & $27.99 \pm 9.45$ & $<0.001$ \\
\hline PBF & $33.82 \pm 9.58$ & $33.09 \pm 9.56$ & $37.69 \pm 8.73$ & $<0.001$ \\
\hline $\operatorname{VFA}\left(\mathrm{cm}^{2}\right)$ & $121.93 \pm 51.83$ & $118.18 \pm 51.29$ & $141.71 \pm 50.13$ & $<0.001$ \\
\hline SLM (kg) & $3085(41.90)$ & $45.49 \pm 9.07$ & $42.82 \pm 8.40$ & $<0.001$ \\
\hline VAI (male) & $4.44 \pm 3.60$ & $4.37 \pm 3.60$ & $4.84 \pm 3.60$ & $<0.001$ \\
\hline VAI (female) & $6.54 \pm 5.31$ & $6.43 \pm 5.30$ & $7.13 \pm 5.30$ & $<0.001$ \\
\hline AIP & $0.97 \pm 0.65$ & $0.0 .94 \pm 0.65$ & $1.07 \pm 0.61$ & $<0.001$ \\
\hline TG (mg/dl) & $136.61 \pm 84.00$ & $134.16 \pm 83.05$ & $149.57 \pm 87.78$ & $<0.001$ \\
\hline HDL-C (mg/dl) & $46.40 \pm 11.35$ & $46.41 \pm 11.36$ & $46.36 \pm 11.27$ & 0.555 \\
\hline LDL-C (mg/dl) & $101.86 \pm 25.42$ & $101.55 \pm 25.19$ & $103.51 \pm 26.55$ & 0.007 \\
\hline $\mathrm{T}-\mathrm{C}(\mathrm{mg} / \mathrm{dl})$ & $185.11 \pm 38.10$ & $184.54 \pm 37.64$ & $188.13 \pm 40.24$ & 0.003 \\
\hline FBS (mg/dl) & $96.94 \pm 30.05$ & $94.83 \pm 27.13$ & $108.11 \pm 40.47$ & $<0.001$ \\
\hline ALP (UI/L) & $197.11 \pm 61.02$ & $194.46 \pm 56.52$ & $211.13 \pm 79.29$ & $<0.001$ \\
\hline
\end{tabular}




\begin{tabular}{|c|c|c|c|c|}
\hline Parameters & $\begin{array}{l}\text { Total } \\
(n=7362)\end{array}$ & $\begin{array}{l}\text { Non-CVDs } \\
(n=6191)\end{array}$ & $\begin{array}{l}\text { CVDs } \\
(n=1171)\end{array}$ & P value \\
\hline AST (UI/L) & $21.43 \pm 8.95$ & $21.47 \pm 9.06$ & $21.20 \pm 8.36$ & 0.325 \\
\hline ALT (UI/L) & $24.70 \pm 14.28$ & $24.84 \pm 14.53$ & $23.94 \pm 12.88$ & 0.045 \\
\hline GGT (UI/L) & $24.30 \pm 18.89$ & $23.86 \pm 18.58$ & $26.63 \pm 20.30$ & $<0.001$ \\
\hline Hypertension, n (\%) & 1351 (18.35) & $411(6.64)$ & $940(80.27)$ & $<0.001$ \\
\hline Dyslipidemia, n (\%) & 3085 (41.90) & $2567(41.46)$ & $518(44.24)$ & 0.078 \\
\hline MetS, n (\%) & 1891 (25.69) & $1261(20.37)$ & $630(53.80)$ & $<0.001$ \\
\hline \multicolumn{5}{|c|}{$\begin{array}{l}\text { BMI: Body mass index; WHR: Waist hip ratio; WC: Waist circumference; BFM: Body fat mass ; PBF: } \\
\text { Percent body fat; VFA: Visceral fat area; SLM: Skeletal muscle mass; VAl: Visceral Adiposity Index; } \\
\text { AIP: Atherogenic index of plasma ; TG: Triglycerides; HDL-C: high-density lipoprotein cholesterol ; LDL } \\
\text { C: Ligh-density lipoprotein cholesterol ; T- C: Total cholesterol; FBS: Fasting blood sugar; ALP: Alkaline } \\
\text { phosphatase; AST: Aspartate transaminase; ALT: Alanine aminotransferase; GGT: Gamma-glutamyl } \\
\text { transferase ; CVDs: Cardiovascular diseases, MetS: Metabolic syndrome }\end{array}$} \\
\hline
\end{tabular}

The mean BMI in the first to third tertile of VAl were $25.82 \pm 4.70,27.75 \pm 4.77$ and $28.40 \pm 4.17 \mathrm{~kg} / \mathrm{m}^{2}$, respectively $(P<0.001)$ in males; also BMI has increased significantly with increasing VAl in females. The mean FBS in the first to third tertile of VAl were $91.33 \pm 24.40,95.94 \pm 28.58$ and $102.54 \pm 34.45 \mathrm{mg} / \mathrm{dl}$, respectively $(P<0.001)$ in males; also FBS has increased significantly with increasing VAl in females. There were significant differences in anthropometric indices including WC and WHR and lipid profile (TG, HDL-C, LDL, TC) between VAl tertiles $(P<0.001)$ in males and females. Participants with higher VAl had significantly more hypertension, dyslipidemia, MetS and CVDs compared to those with low VAI (Table 2). 
Table 2

Baseline Characteristics according to tertiles of visceral adiposity index (VAI) and atherogenic index of plasma (AIP) among by sex

\begin{tabular}{|c|c|c|c|c|c|c|c|c|}
\hline \multirow[t]{4}{*}{ Parameters } & \multicolumn{8}{|c|}{ Tertiles of Visceral Adiposity Index } \\
\hline & \multicolumn{4}{|l|}{ Male } & \multicolumn{4}{|l|}{ Female } \\
\hline & \multirow{2}{*}{$\begin{array}{l}\text { T1 } \\
(n= \\
684)\end{array}$} & \multirow{2}{*}{$\begin{array}{l}\text { T2 } \\
(n= \\
1228)\end{array}$} & \multirow{2}{*}{$\begin{array}{l}\text { T3 } \\
(n= \\
1508)\end{array}$} & \multirow{2}{*}{$\begin{array}{l}P \\
\text { value }\end{array}$} & \multirow{2}{*}{$\begin{array}{l}\mathrm{T} 1 \\
(\mathrm{n}= \\
1367)\end{array}$} & \multirow{2}{*}{$\begin{array}{l}\text { T2 } \\
(n= \\
1532)\end{array}$} & \multirow{2}{*}{$\begin{array}{l}\text { T3 } \\
(n= \\
1043)\end{array}$} & \multirow{2}{*}{$\begin{array}{l}P \\
\text { value }\end{array}$} \\
\hline & & & & & & & & \\
\hline Age (year) & $\begin{array}{l}46.36 \pm \\
8.39\end{array}$ & $\begin{array}{l}47.52 \pm \\
8.39\end{array}$ & $\begin{array}{l}47.52 \pm \\
8.19\end{array}$ & $\begin{array}{l}< \\
0.001\end{array}$ & $\begin{array}{l}45.82 \pm \\
8.31\end{array}$ & $\begin{array}{l}46.99 \pm \\
8.37\end{array}$ & $\begin{array}{l}47.58 \pm \\
8.28\end{array}$ & $\begin{array}{l}< \\
0.001\end{array}$ \\
\hline $\mathrm{BMI}\left(\mathrm{kg} / \mathrm{m}^{2}\right)$ & $\begin{array}{l}25.82 \pm \\
4.70\end{array}$ & $\begin{array}{l}27.75 \pm \\
4.77\end{array}$ & $\begin{array}{l}28.40 \pm \\
4.17\end{array}$ & $<.001$ & $\begin{array}{l}24.80 \pm \\
4.43\end{array}$ & $\begin{array}{l}26.89 \pm \\
4.81\end{array}$ & $\begin{array}{l}28.22 \pm \\
4.40\end{array}$ & <. 001 \\
\hline WHR & $\begin{array}{l}0.91 \pm \\
0.06\end{array}$ & $\begin{array}{l}0.94 \pm \\
0.06\end{array}$ & $\begin{array}{l}0.95 \pm \\
0.06\end{array}$ & $<.001$ & $\begin{array}{l}0.90 \pm \\
0.06\end{array}$ & $\begin{array}{l}0.93 \pm \\
0.06\end{array}$ & $\begin{array}{l}0.95 \pm \\
0.06\end{array}$ & $\begin{array}{l}< \\
0.001\end{array}$ \\
\hline WC (cm) & $\begin{array}{l}92.63 \pm \\
10.98\end{array}$ & $\begin{array}{l}97.62 \pm \\
10.49\end{array}$ & $\begin{array}{l}99.62 \pm \\
9.13\end{array}$ & $<.001$ & $\begin{array}{l}90.01 \pm \\
10.79\end{array}$ & $\begin{array}{l}95.26 \pm \\
10.72\end{array}$ & $\begin{array}{l}99.12 \pm \\
9.66\end{array}$ & $\begin{array}{l}< \\
0.001\end{array}$ \\
\hline $\mathrm{TG}(\mathrm{mg} / \mathrm{dl})$ & $\begin{array}{l}70.50 \pm \\
18.93\end{array}$ & $\begin{array}{l}114.35 \\
\pm 25.55\end{array}$ & $\begin{array}{l}213.83 \\
\pm 96.73\end{array}$ & $<.001$ & $\begin{array}{l}56.08 \pm \\
14.50\end{array}$ & $\begin{array}{l}88.39 \pm \\
19.74\end{array}$ & $\begin{array}{l}177.79 \\
\pm 88.22\end{array}$ & $\begin{array}{l}< \\
0.001\end{array}$ \\
\hline $\begin{array}{l}\mathrm{HDL-C} \\
(\mathrm{mg} / \mathrm{dl})\end{array}$ & $\begin{array}{l}55.89 \pm \\
11.31\end{array}$ & $\begin{array}{l}46.49 \pm \\
8.60\end{array}$ & $\begin{array}{l}38.67 \pm \\
7.54\end{array}$ & $<001$ & $\begin{array}{l}60.64 \pm \\
12.00\end{array}$ & $\begin{array}{l}50.93 \pm \\
9.58\end{array}$ & $\begin{array}{l}41.28 \pm \\
8.42\end{array}$ & $\begin{array}{l}< \\
0.001\end{array}$ \\
\hline $\begin{array}{l}\text { LDL-C } \\
(\mathrm{mg} / \mathrm{dl})\end{array}$ & $\begin{array}{l}92.85 \pm \\
23.03\end{array}$ & $\begin{array}{l}102.93 \\
\pm 24.56\end{array}$ & $\begin{array}{l}107.95 \\
\pm 26.14\end{array}$ & $<.001$ & $\begin{array}{l}88.63 \pm \\
21.54\end{array}$ & $\begin{array}{l}97.52 \pm \\
24.35\end{array}$ & $\begin{array}{l}106.69 \\
\pm 25.37\end{array}$ & $\begin{array}{l}<.001 \\
0.001\end{array}$ \\
\hline $\mathrm{T}-\mathrm{C}(\mathrm{mg} / \mathrm{dl})$ & $\begin{array}{l}173.74 \\
\pm 34.56\end{array}$ & $\begin{array}{l}184.76 \\
\pm 36.43\end{array}$ & $\begin{array}{l}194.63 \\
\pm 39.97\end{array}$ & $<.001$ & $\begin{array}{l}170.48 \\
\pm 32.71\end{array}$ & $\begin{array}{l}178.15 \\
\pm 36.26\end{array}$ & $\begin{array}{l}191.61 \\
\pm 38.57\end{array}$ & $\begin{array}{l}< \\
0.001\end{array}$ \\
\hline FBS $(\mathrm{mg} / \mathrm{dl})$ & $\begin{array}{l}91.33 \pm \\
24.40\end{array}$ & $\begin{array}{l}95.94 \pm \\
28.58\end{array}$ & $\begin{array}{l}102.54 \\
\pm 34.45\end{array}$ & $<.001$ & $\begin{array}{l}90.49 \pm \\
23.55\end{array}$ & $\begin{array}{l}92.94 \pm \\
25.79\end{array}$ & $\begin{array}{l}100.31 \\
\pm 32.70\end{array}$ & $\begin{array}{l}< \\
0.001\end{array}$ \\
\hline $\begin{array}{l}\text { Hypertension, } \\
\mathrm{n}(\%)\end{array}$ & $\begin{array}{l}253 \\
(18.73)\end{array}$ & $\begin{array}{l}558 \\
(41.30)\end{array}$ & $\begin{array}{l}540 \\
(39.97)\end{array}$ & $<.001$ & $\begin{array}{l}78 \\
(5.77)\end{array}$ & $\begin{array}{l}368 \\
(27.24)\end{array}$ & $\begin{array}{l}905 \\
(66.99)\end{array}$ & $\begin{array}{l}<.001 \\
0.001\end{array}$ \\
\hline $\begin{array}{l}\text { Dyslipidemia, } \\
\text { n (\%) }\end{array}$ & $\begin{array}{l}169 \\
(5.48)\end{array}$ & $\begin{array}{l}824 \\
(26.71)\end{array}$ & $\begin{array}{l}2092 \\
(67.81)\end{array}$ & $\begin{array}{l}<.001 \\
0.001\end{array}$ & $\begin{array}{l}29 \\
(0.94)\end{array}$ & $\begin{array}{l}362 \\
(11.73)\end{array}$ & $\begin{array}{l}2694 \\
(87.33)\end{array}$ & $\dot{0.001}$ \\
\hline MetS, n (\%) & $\begin{array}{l}269 \\
(14.23)\end{array}$ & $\begin{array}{l}572 \\
(30.25)\end{array}$ & $\begin{array}{l}1050 \\
(55.53)\end{array}$ & $<.001$ & $\begin{array}{l}88 \\
(4.65)\end{array}$ & $\begin{array}{l}355 \\
(18.77)\end{array}$ & $\begin{array}{l}1448 \\
(76.57)\end{array}$ & $\begin{array}{l}< \\
0.001\end{array}$ \\
\hline CVD, n (\%) & $\begin{array}{l}235 \\
(20.07)\end{array}$ & $\begin{array}{l}471 \\
(40.22)\end{array}$ & $\begin{array}{l}465 \\
(39.71)\end{array}$ & $<001$ & $\begin{array}{l}70 \\
(5.98)\end{array}$ & $\begin{array}{l}336 \\
(28.69)\end{array}$ & $\begin{array}{l}765 \\
(65.33)\end{array}$ & $\begin{array}{l}< \\
0.001\end{array}$ \\
\hline \multicolumn{9}{|c|}{ Tertiles of Atherogenic index of plasma } \\
\hline & $\begin{array}{l}T 1(n= \\
866)\end{array}$ & $\begin{array}{l}\text { T2 }(\mathrm{n}= \\
1119)\end{array}$ & $\begin{array}{l}\text { T3 }(n= \\
1435)\end{array}$ & $\begin{array}{l}P \\
\text { value }\end{array}$ & $\begin{array}{l}T 1(n= \\
1588)\end{array}$ & $\begin{array}{l}T 2(n= \\
1335)\end{array}$ & $\begin{array}{l}\text { T3 }(n= \\
1019)\end{array}$ & $\begin{array}{l}P \\
\text { value }\end{array}$ \\
\hline
\end{tabular}




\begin{tabular}{|c|c|c|c|c|c|c|c|c|}
\hline \multirow[t]{3}{*}{ Parameters } & \multicolumn{8}{|c|}{ Tertiles of Visceral Adiposity Index } \\
\hline & \multicolumn{4}{|l|}{ Male } & \multicolumn{4}{|l|}{ Female } \\
\hline & $\begin{array}{l}T 1 \\
(n= \\
684)\end{array}$ & $\begin{array}{l}\text { T2 } \\
(n= \\
1228)\end{array}$ & $\begin{array}{l}\text { T3 } \\
(n= \\
1508)\end{array}$ & $\begin{array}{l}P \\
\text { value }\end{array}$ & $\begin{array}{l}\text { T1 } \\
(n= \\
1367)\end{array}$ & $\begin{array}{l}\text { T2 } \\
(n= \\
1532)\end{array}$ & $\begin{array}{l}\text { T3 } \\
(n= \\
1043)\end{array}$ & $\begin{array}{l}P \\
\text { value }\end{array}$ \\
\hline Age (year) & $\begin{array}{l}46.98 \pm \\
8.37\end{array}$ & $\begin{array}{l}46.81 \pm \\
8.23\end{array}$ & $\begin{array}{l}46.42 \pm \\
7.88\end{array}$ & $\begin{array}{l}< \\
0.001\end{array}$ & $\begin{array}{l}46.56 \pm \\
8.52\end{array}$ & $\begin{array}{l}48.07 \pm \\
8.35\end{array}$ & $\begin{array}{l}48.73 \pm \\
8.41\end{array}$ & $\begin{array}{l}< \\
0.001\end{array}$ \\
\hline $\mathrm{BMI}\left(\mathrm{kg} / \mathrm{m}^{2}\right)$ & $\begin{array}{l}23.99 \pm \\
388\end{array}$ & $\begin{array}{l}26.07 \pm \\
3.89\end{array}$ & $\begin{array}{l}27.64 \pm \\
3.65\end{array}$ & $\begin{array}{l}< \\
0.001\end{array}$ & $\begin{array}{l}27.04 \pm \\
4.81\end{array}$ & $\begin{array}{l}29.29 \pm \\
4.83\end{array}$ & $\begin{array}{l}19.74 \pm \\
4.53\end{array}$ & $\begin{array}{l}< \\
0.001\end{array}$ \\
\hline WHR & $\begin{array}{l}0.90 \pm \\
0.06\end{array}$ & $\begin{array}{l}0.94 \pm \\
0.06\end{array}$ & $\begin{array}{l}0.96 \pm \\
0.06\end{array}$ & $\hat{0}_{0.001}$ & $\begin{array}{l}0.93 \pm \\
0.06\end{array}$ & $\begin{array}{l}0.95 \pm \\
0.05\end{array}$ & $\begin{array}{l}0.96 \pm \\
0.05\end{array}$ & $\begin{array}{l}< \\
0.001\end{array}$ \\
\hline WC (cm) & $\begin{array}{l}91.54 \pm \\
9.66\end{array}$ & $\begin{array}{l}95.58 \pm \\
9.61\end{array}$ & $\begin{array}{l}98.45 \pm \\
8.67\end{array}$ & $\begin{array}{l}< \\
0.001\end{array}$ & $\begin{array}{l}95.12 \pm \\
11.63\end{array}$ & $\begin{array}{l}99.44 \pm \\
10.89\end{array}$ & $\begin{array}{l}100.36 \\
\pm 9.86\end{array}$ & $\begin{array}{l}< \\
0.001\end{array}$ \\
\hline $\mathrm{TG}(\mathrm{mg} / \mathrm{dl})$ & $\begin{array}{l}70.73 \pm \\
18.62\end{array}$ & $\begin{array}{l}112.96 \\
\pm 22.88\end{array}$ & $\begin{array}{l}216.34 \\
\pm 95.55\end{array}$ & $\begin{array}{l}<.001 \\
0.001\end{array}$ & $\begin{array}{l}75.75 \pm \\
20.71\end{array}$ & $\begin{array}{l}123.99 \\
\pm 25.59\end{array}$ & $\begin{array}{l}217.64 \\
\pm 99.69\end{array}$ & $\begin{array}{l}< \\
0.001\end{array}$ \\
\hline $\begin{array}{l}\mathrm{HDL-C} \\
(\mathrm{mg} / \mathrm{dl})\end{array}$ & $\begin{array}{l}51.67 \pm \\
10.68\end{array}$ & $\begin{array}{l}43.21 \pm \\
7.23\end{array}$ & $\begin{array}{l}36.80 \pm \\
6.89\end{array}$ & $<0.001$ & $\begin{array}{l}56.66 \pm \\
11.04\end{array}$ & $\begin{array}{l}48.06 \pm \\
8.61\end{array}$ & $\begin{array}{l}40.78 \pm \\
7.69\end{array}$ & $\begin{array}{l}< \\
0.001\end{array}$ \\
\hline $\begin{array}{l}\mathrm{LDL}-\mathrm{C} \\
(\mathrm{mg} / \mathrm{dl})\end{array}$ & $\begin{array}{l}91.74 \pm \\
23.69\end{array}$ & $\begin{array}{l}101.39 \\
\pm 23.69\end{array}$ & $\begin{array}{l}106.02 \\
\pm 24.59\end{array}$ & $\begin{array}{l}<.001 \\
0.001\end{array}$ & $\begin{array}{l}94.83 \pm \\
23.52\end{array}$ & $\begin{array}{l}105.86 \\
\pm 24.33\end{array}$ & $\begin{array}{l}110.85 \\
\pm 28.29\end{array}$ & $\begin{array}{l}< \\
0.001\end{array}$ \\
\hline $\mathrm{T}-\mathrm{C}(\mathrm{mg} / \mathrm{dl})$ & $\begin{array}{l}169.27 \\
\pm 34.17\end{array}$ & $\begin{array}{l}178.96 \\
\pm 34.12\end{array}$ & $\begin{array}{l}190.30 \\
\pm 37.54\end{array}$ & $\begin{array}{l}<.001 \\
0.001\end{array}$ & $\begin{array}{l}177.58 \\
\pm 35.34\end{array}$ & $\begin{array}{l}191.42 \\
\pm 36.62\end{array}$ & $\begin{array}{l}201.46 \\
\pm 42.75\end{array}$ & $\begin{array}{l}< \\
0.001\end{array}$ \\
\hline FBS (mg/dl) & $\begin{array}{l}92.64 \pm \\
29.54\end{array}$ & $\begin{array}{l}96.24 \pm \\
29.13\end{array}$ & $\begin{array}{l}100.56 \\
\pm 32.40\end{array}$ & $\begin{array}{l}<.001 \\
0.001\end{array}$ & $\begin{array}{l}91.39 \pm \\
22.17\end{array}$ & $\begin{array}{l}97.38 \pm \\
29.64\end{array}$ & $\begin{array}{l}104.34 \\
\pm 36.36\end{array}$ & $\begin{array}{l}< \\
0.001\end{array}$ \\
\hline $\begin{array}{l}\text { Hypertension, } \\
\mathrm{n}(\%)\end{array}$ & $\begin{array}{l}84 \\
(9.70)\end{array}$ & $\begin{array}{l}181 \\
(16.18)\end{array}$ & $\begin{array}{l}230 \\
(16.03)\end{array}$ & $\begin{array}{l}<.001 \\
0.001\end{array}$ & $\begin{array}{l}241 \\
(15.18)\end{array}$ & $\begin{array}{l}327 \\
(24.49)\end{array}$ & $\begin{array}{l}288 \\
(28.26)\end{array}$ & $\begin{array}{l}< \\
0.001\end{array}$ \\
\hline $\begin{array}{l}\text { Dyslipidemia, } \\
\text { n (\%) }\end{array}$ & $\begin{array}{l}108 \\
(12.47)\end{array}$ & $\begin{array}{l}424 \\
(37.89)\end{array}$ & $\begin{array}{l}1248 \\
(86.97)\end{array}$ & $\begin{array}{l}< \\
0.001\end{array}$ & $\begin{array}{l}134 \\
(8.44)\end{array}$ & $\begin{array}{l}355 \\
(26.59)\end{array}$ & $\begin{array}{l}816 \\
(80.08)\end{array}$ & $\begin{array}{l}< \\
0.001\end{array}$ \\
\hline MetS, n (\%) & $\begin{array}{l}100 \\
(11.55)\end{array}$ & $\begin{array}{l}219 \\
(19.57)\end{array}$ & $\begin{array}{l}530 \\
(36.93)\end{array}$ & $\hat{0}_{0.001}$ & $\begin{array}{l}238 \\
(14.99)\end{array}$ & $\begin{array}{l}326 \\
(24.42)\end{array}$ & $\begin{array}{l}478 \\
(46.91)\end{array}$ & $\begin{array}{l}<.001 \\
0.001\end{array}$ \\
\hline CVD, n (\%) & $\begin{array}{l}78 \\
(9.01)\end{array}$ & $\begin{array}{l}128 \\
(11.44)\end{array}$ & $\begin{array}{l}178 \\
(12.40)\end{array}$ & $\begin{array}{l}< \\
0.001\end{array}$ & $\begin{array}{l}235 \\
(14.80)\end{array}$ & $\begin{array}{l}287 \\
(21.50)\end{array}$ & $\begin{array}{l}265 \\
(26.02)\end{array}$ & $\begin{array}{l}< \\
0.001\end{array}$ \\
\hline $\begin{array}{l}\text { BMI: Body ma } \\
\text { TG: Triglycerid } \\
\text { cholesterol ; T- } \\
\text { CVDs: Cardiov }\end{array}$ & cular dis & & atip & drom & mforan & VD: Carc & acoular & $\begin{array}{l}\text { eases; } \\
\text { sterol; }\end{array}$ \\
\hline
\end{tabular}

The mean WHR in the first to third tertile of AIP were $0.90 \pm 0.06,0.94 \pm 0.06$ and $0.96 \pm 0.06$, respectively $(P<0.001)$ in males. There were significant differences in anthropometric indices and lipid profile between AIP tertiles $(P<0.001)$. Also, participants with higher AIP had significantly more hypertension, dyslipidemia MetS and CVDs compared to those with low AIP (Table 2). 
Univariate logistic regression analysis showed that increase of AIP level was associated with an increased risk of CVDs. Risk of CVDs in the second tertile and third tertile AIP was 1.39 (95\% Cl: 1.19, $1.63)$ and $1.51(95 \% \mathrm{Cl}: 1.28,1.76)$ times higher than the first tertile, respectively. In multiple logistic regression analysis and after adjusting for age and sex, an increase of AIP and VAl level was significantly associated with an increased risk of CVDs (Model2). Moreover, model 3 shows that after adjusting for age, sex, BMI and physical activity the risk of CVDs has increased in the third tertile AIP 1.40 (95\% Cl: $1.19,1.66)$ times compared to first tertile; and in the third tertile VAl has increased 1.52 ( $95 \% \mathrm{Cl}: 1.25$, 1.83) times than first tertile (Table 3 ).

Table 3

Association of atherogenic index of plasma and visceral adiposity index with cardiovascular diseases (logistic regression analysis)

\begin{tabular}{|c|c|c|c|c|c|c|}
\hline \multirow[t]{2}{*}{ Variables } & \multicolumn{2}{|l|}{ Model 1} & \multicolumn{2}{|l|}{ Model 2} & \multicolumn{2}{|l|}{ Model 3} \\
\hline & OR (95\% Cl) & $P$ value & OR (95\% Cl) & $P$ value & OR (95\% Cl) & $P$ value \\
\hline AIP : T1 & 1.00 (Reference) & & 1.00 (Reference) & & 1.00 (Reference) & \\
\hline T2 & $1.39(1.19,1.63)$ & $<0.001$ & $1.42(1.20,1.69)$ & $<0.001$ & $1.22(1.02,1.45)$ & 0.026 \\
\hline T3 & $1.51(1.28,1.76)$ & $<0.001$ & $1.72(1.45,2.04)$ & $<0.001$ & $1.40(1.19,1.66)$ & $<0.001$ \\
\hline VAl : T1 & 1.00 (Reference) & & 1.00 (Reference) & & 1.00 (Reference) & \\
\hline T2 & $1.48(1.26,1.74)$ & $<0.001$ & $1.56(1.32,1.86)$ & $<0.001$ & $1.28(1.06,1.53)$ & 0.009 \\
\hline T3 & $1.80(1.54,2.11)$ & $<0.001$ & $2.08(1.76,2.47)$ & $<0.001$ & $1.52(1.25,1.83)$ & $<0.001$ \\
\hline \multicolumn{7}{|c|}{$\begin{array}{l}\text { Model 1: Unadjusted; Model 2: Adjusted for age and sex, Model 3: Adjusted for age, sex, BMI, physical } \\
\text { activity }\end{array}$} \\
\hline \multicolumn{7}{|c|}{ AIP: Atherogenic index of plasma, VAI: Visceral Adiposity Index, T: tertile } \\
\hline
\end{tabular}

\section{Discussion}

Findings of this study showed that there is a positive association between AIP and VAI with the risk of CVDs in adults, and this association remained significant after adjusting for potential confounders. In addition, males and females with higher VAI and AIP had significantly more hypertension, dyslipidemia, MetS and CVDs compared to those with low VAI and AIP. The higher anthropometric indices, lipid profile and FBS were also associated with higher VAI and AIP.

The mean of AIP and VAl in our study was somewhat higher than other studies $[13,15,16]$, which could be due to the studied age group (35-65 years) and the high prevalence of obesity and overweight in participants. Moreover, mean VAI was higher in female participants compared to males; higher VAl was associated with higher prevalence of CVDs in both males (first tertile: $20 \%$ vs. third tertile: $39.71 \%$ ) and 
females (first tertile: $5.98 \%$ vs. third tertile: $65.33 \%$ ). However, in this study, although the male BMI was higher compared to the female, the females VAI was higher than the males.

Our findings show that increasing VAI can increase the risk of CVDs by about $52 \%$. The association between VAl and heart disease and its risk factors has been reported in previous studies; In a cohort study in Greece has been showed that VAI were independently associated with CVDs [7]. Moreover, our findings showed participants with higher VAI had significantly more hypertension, dyslipidemia, T2DM and MetS compared to those with low VAI. Similar to our findings, a meta-analysis study done in 2019 demonstrated that VAl can be an independent predictor to T2DM in Asian populations [24]. In addition, the predictive power of VAl using ROC curve analysis has shown the accuracy and reliability of VAI for predicting T2DM, hypertension, and MetS $[9,11,25]$. Overall, this finding is important because these factors are risk factors for CVDs; and can be prevented disease by controlling risk factors.

Our study demonstrated, high anthropometric indices (BMI, WHR and WC) associated with higher CVDs. The BMI was $27.2 \mathrm{~kg} / \mathrm{m}^{2}$ in non-CVDs and $28.7 \mathrm{~kg} / \mathrm{m}^{2}$ in CVDs group, WHR was 0.93 and 0.95 among non-CVDs and CVDs group, respectively. Zhu and et al. presented, an increase in anthropometric indices (BMI and WC) and liver enzymes (ALT and GGT) and LDL-C was associated with an increase in AIP levels [26], which is consistent with the findings of our study. Moreover, we found that the average VFA in CVDs patients was significantly higher than in non- CVDs (118.18 vs. $\left.141.71 \mathrm{~cm}^{2}\right)$, which this is consistent with the findings of a study by Bo et al [27]. The mechanism of this association can be expressed in this way that visceral fat is mainly depleted by the portal venous system and then discharged into the liver and leading to insulin resistance in addition, excess free fatty acids (FFA) may cause the enhancement of lipid synthesis and gluconeogenesis, as well as insulin resistance, resulting in hyperlipidemia, glucose intolerance, hypertension, and finally atherosclerosis [28].

Our findings showed that high AIP can increase the risk of CVDs by $40 \%$. A positive association between AIP and CVDs has already been observed in different populations such as postmenopausal women and staff $[12,27]$. Moreover, AIP has a positive association with non-alcoholic fatty liver disease (NAFLD), advanced subclinical coronary artery disease (CAD), ischemic stroke, atherosclerosis, MetS and obesity; and is a useful and reliable marker for predicting these diseases [6, 26, 29-31]. A study on Malaysian showed that among lipid profile, AIP was more positively correlated with TG and more negatively correlated with HDL-C, thus they consider AIP to be the strongest marker in predicting CVDs risk over other indices [27]. Other research has shown, AIP was the most sensitive marker compared with other atherogenic indices including (TC/HDL-C), (LDL-C/HDL-C), and atherogenic coefficient (TC-HDL-C/HDL-C) $[32,33]$. Isolated elevation in TG increases the CVDs risk but these effects may be balanced by cardioprotective lipoprotein of HDL-C [34].

Following the industrialization of societies and the rapid growth of urbanization, the physical activity has been reduced, which has led to an increase in general and central obesity, increased pre-diabetes, T2DM, hypercholesterolemia, hypertension and MetS [35-37]. Considering to the limitations of older indices such as BMI and WC- mentioned in previous studies[38-40] - and the high prevalence of morbidity and 
mortality related to CVDs and need to a suitable tool to screening it; VAI and AIP can be a useful, inexpensive and accurate tool for predicting NCDs, especially CVDs [7].

The most important strength of this study was the use of RaNCD prospective study data. This is the first study about the association of AIP and VAI with CVDs on a large population with Kurdish ethnicity. The large sample size is one of the positive points of this study. The main limitation of this study is its crosssectional nature, which limits the causal inference of the observed associations.

\section{Conclusion}

Findings of this study demonstrated that there is a positive association between AIP and VAI with the risk of CVDs in adults, and this association remained significant after adjust for potential confounders. Therefore, AIP and VAI can be used as markers to predict of CVDs.

\section{Abbreviations}

AIP

Atherogenic index of plasma; BMI:Body mass index; CAD:Coronary artery disease; FFA:free fatty acids, FBS:Fasting blood sugar; GBD:Global burden of diseases; NCDs:Non-Communicable disease; CVDs:Cardiovascular diseases; HDL-C:High density lipoprotein; Ml:Myocardial infraction; ALP:Alkaline phosphatase; AST:Aspartate transaminase; ALT:Alanine aminotransferase; GGT:Gamma-glutamyl transferase ; CVDs:Cardiovascular diseases, MetS:Metabolic syndrome; PBF:Percent body fat; VFA:Visceral fat area; SLM:Skeletal muscle mass; VAI:Visceral Adiposity Index; NAFLD:Non-alcoholic fatty liver disease; RaNCD:Ravansar non-communicable diseases; PERSIAN:Prospective epidemiologic research of IRAN; VLDL:Very-low-density lipoprotein; VAI:Visceral Adiposity index; LDL-C:Low density lipoprotein; TG:Triglyceride; WC:Waist circumference.

\section{Declarations}

\section{Authors' contributions}

$\mathrm{BH}$ and YP generated the initial idea for the study, FN and ESH designed the experiments, MD and RSF carried out all analyses and visualization of the results. MD and NM drafted the manuscript. All authors provided critical input into the interpretation of the results, revisions to the manuscript and approved the final draft.

\section{Funding}

This study was supported by the Kermanshah University of Medical Sciences, Kermanshah, Iran (grant number 990607).

\section{Acknowledgments}


The authors thank the PERSIAN cohort Study collaborators and of Kermanshah University of Medical Sciences.

\section{Ethics approval and consent to participate}

The Research and Technology Deputy and the Ethical Committee of Kermanshah University of Medical Sciences have approved the study protocol (Ethical Number: KUMS.REC.1399.639). Participants were provided with oral and written informed consent. Written informed consent was obtained from all subjects prior to enrollment in the study.

\section{Availability of data and materials}

The data analyzed in the study are available from the corresponding author on reasonable request.

\section{Consent for publication}

N/A

\section{Conflict of interest}

The authors declare no conflicts of interest.

\section{References}

1. Roth GA, et al. Global, regional, and national age-sex-specific mortality for 282 causes of death in 195 countries and territories, 1980-2017: a systematic analysis for the Global Burden of Disease Study 2017. The Lancet. 2018;392(10159):1736-88. https://doi.org/ 10.1016/S01406736(18)32203-7.

2. Sadeghi M, Haghdoost AA, Bahrampour A, Dehghani M. Modeling the burden of cardiovascular diseases in Iran from 2005 to 2025: The impact of demographic changes. Iran J Public Health. 2017;46(4):506.

3. Organization WH. Group ISoHW. 2003 World Health Organization (WHO)/International Society of Hypertension (ISH) statement on management of hypertension. Journal of hypertension. 2003;21(11):1983-92.

4. Murray CJ, et al. Effectiveness and costs of interventions to lower systolic blood pressure and cholesterol: a global and regional analysis on reduction of cardiovascular-disease risk. The Lancet. 2003;361(9359):717-25. https://doi.org/ 10.1016/S0140-6736(03)12655-4.

5. Darbandi M, Pasdar Y, Moradi S, Mohamed HJJ, Hamzeh B, Salimi Y. Discriminatory Capacity of Anthropometric Indices for Cardiovascular Disease in Adults: A Systematic Review and MetaAnalysis. Prev Chronic Dis. 2020;17:E131. https://doi.org/ 10.5888/pcd17.200112.

6. Cure $E$, et al. Atherogenic index of plasma: a useful marker for subclinical atherosclerosis in ankylosing spondylitis. Clin Rheumatol. 2018;37(5):1273-80. https://doi.org/ 10.1007/s10067-018- 
4027-0.

7. Kouli G-M, et al. Visceral adiposity index and 10-year cardiovascular disease incidence: the ATTICA study. Nutr Metab Cardiovasc Dis. 2017;27(10):881-9. https://doi.org/10.1016/j.numecd.2017.06.015.

8. Amato MC, et al. Visceral Adiposity Index: a reliable indicator of visceral fat function associated with cardiometabolic risk. Diabetes Care. 2010;33(4):920-2. https://doi.org/ 10.2337/dc09-1825.

9. Baveicy K, Mostafaei S, Darbandi M, Hamzeh B, Najafi F, Pasdar Y. Predicting Metabolic Syndrome by Visceral Adiposity Index, Body Roundness Index and a Body Shape Index in Adults: A CrossSectional Study from the Iranian RaNCD Cohort Data. DDiabetes Metab Syndr Obes. 2020;13:879. https://doi.org/ 10.2147/DMSO.S238153.

10. Nusrianto R, et al. Visceral adiposity index and lipid accumulation product as a predictor of type 2 diabetes mellitus: the Bogor cohort study of non-communicable diseases risk factors. Diabetes Res Clin Pract. 2019;155:107798. https://doi.org/ 10.1016/j.diabres.2019.107798.

11. Zhang Z, et al. Visceral adiposity index (VAl), a powerful predictor of incident hypertension in prehypertensives. Intern Emerg Med. 2018;13(4):509-16. https://doi.org/ 10.1007/s11739-018-18368.

12. Nansseu JRN, Moor VJA, Nouaga MED, Zing-Awona B, Tchanana G, Ketcha A. Atherogenic index of plasma and risk of cardiovascular disease among Cameroonian postmenopausal women. Lipids Health Dis. 2016;15(1):1-5. 10.1186/s12944-016-0222-7.

13. Niroumand S, et al. Atherogenic Index of Plasma (AIP): A marker of cardiovascular disease. Med J Islam Repub Iran. 2015;29:240. 10.1016/j.arcmed.2019.08.009.

14. Nwagha U, Ikekpeazu E, Ejezie F, Neboh E, Maduka I. Atherogenic index of plasma as useful predictor of cardiovascular risk among postmenopausal women in Enugu, Nigeria. Afr Health Sci. 2010;10(3).

15. Javardi MSM, Madani Z, Movahedi A, Karandish M, Abbasi B. The correlation between dietary fat quality indices and lipid profile with Atherogenic index of plasma in obese and non-obese volunteers: a cross-sectional descriptive-analytic case-control study. Lipids Health Dis. 2020;19(1):1-9. 10.1186/s12944-020-01387-4.

16. Ebrahimi R, et al. Low level of adiponectin predicts the development of Nonalcoholic fatty liver disease: Is it irrespective to visceral adiposity index, visceral adipose tissue thickness and other obesity indices? Arch Physiol Biochem. 2019:1-8. 10.1080/13813455.2019.1661496.

17. Janghorbani M, Salamat MR, Aminorroaya A, Amini M. Utility of the visceral adiposity index and hypertriglyceridemic waist phenotype for predicting incident hypertension. Endocrinol Metab. 2017;32(2):221-9. https://doi.org/ 10.3803/EnM.2017.32.2.221.

18. Ehsani B, Moslehi N, Mirmiran P, Tehrani FR, Tahmasebinejad Z, Azizi F. A visceral adiposity indexrelated dietary pattern and the cardiometabolic profiles in women with polycystic ovary syndrome. Clin nutr. 2016;35(5):1181-7. https://doi.org/ 10.1016/j.clnu.2015.10.007.

19. Pasdar Y, et al. Cohort profile: Ravansar Non-Communicable Disease cohort study: the first cohort study in a Kurdish population. Int J Epidemiol. 2019;48(3):682-3f. https://doi.org/ 
10.1093/ije/dyy296.

20. Mirzaei M, Asghari-Jafarabadi M, Amini-Sani N, Bakhtari-Aghdam F, Dastgiri S. Psychometric evaluation of a self-reported physical activity questionnarie used in the pilot phase of the AZAR Cohort Study. Health Promot Perspect. 2016;6(3):152. https://doi.org/ 10.15171/hpp.2016.24.

21. Rajati F, et al. Prevalence, awareness, treatment, and control of hypertension and their determinants: Results from the first cohort of non-communicable diseases in a Kurdish settlement. Sci Rep. 2019;9(1):1-10. https://doi.org/ 10.1038/s41598-019-48232-y.

22. Dobiasova M. AIP-atherogenic index of plasma as a significant predictor of cardiovascular risk: from research to practice. Vnitrni lekarstvi. 2006;52(1):64-71.

23. Alberti K, et al. Harmonizing the metabolic syndrome: a joint interim statement of the international diabetes federation task force on epidemiology and prevention; national heart, lung, and blood institute; American heart association; world heart federation; international atherosclerosis society; and international association for the study of obesity. Circulation. 2009;120(16):1640-5. https://doi.org/ 10.1161/CIRCULATIONAHA.109.192644.

24. Nusrianto R, Tahapary DL, Soewondo P. Visceral adiposity index as a predictor for type 2 diabetes mellitus in Asian population: A systematic review. Diabetes Metab Syndr: Clin Res Rev. 2019;13(2):1231-5. https://doi.org/ 10.1016/j.dsx.2019.01.056.

25. Janghorbani M, Amini M. The visceral adiposity index in comparison with easily measurable anthropometric markers did not improve prediction of diabetes. Can J Diabetes. 2016;40(5):393-8. https://doi.org/ 10.1016/j.jcjd.2016.02.008.

26. Qian W, Zheng D, Liu J, Li F, Li Q. Atherogenic index of plasma is a novel predictor of non-alcoholic fatty liver disease in obese participants: a cross-sectional study. Lipids Health Dis. 2018;17(1):1-6. https://doi.org/ 10.1186/s12944-018-0932-0.

27. Bo MS, Cheah WL, Lwin S, Moe Nwe T, Win TT, Aung M. Understanding the relationship between atherogenic index of plasma and cardiovascular disease risk factors among staff of an University in Malaysia. J Nutr Metab. 2018;2018(3):1-6. https://doi.org/ 10.1155/2018/7027624.

28. Bo MS, Cheah WL, Lwin S, Moe Nwe T, Win TT, Aung M. Understanding the relationship between atherogenic index of plasma and cardiovascular disease risk factors among staff of an University in Malaysia. J Nutr Metab. 2018;2018. https://doi.org/ 10.1155/2018/7027624.

29. Zhu X, et al. Atherogenic index of plasma is a novel and better biomarker associated with obesity: a population-based cross-sectional study in China. Lipids Health Dis. 2018;17(1):1-6. https://doi.org/ 10.1186/s12944-018-0686-8.

30. Wang C, et al. Using the Atherogenic Index of Plasma to Estimate the Prevalence of Ischemic Stroke within a General Population in a Rural Area of China. BioMed Research International. 2020;2020. https://doi.org/ 10.1155/2020/7197054.

31. Azarpazhooh MR, Najafi F, Darbandi M, Kiarasi S, Oduyemi T, Spence JD. Triglyceride/High-Density Lipoprotein Cholesterol Ratio: A Clue to Metabolic Syndrome, Insulin Resistance, and Severe Atherosclerosis. Lipids. 2021. https://doi.org/ 10.1002/lipd.12302. 
32. Bhardwaj S, Bhattacharjee J, Bhatnagar M, Tyagi S, Delhi N. Atherogenic index of plasma, castelli risk index and atherogenic coefficient-new parameters in assessing cardiovascular risk. Int J Pharm Biol Sci. 2013;3(3):359-64.

33. Ranjit PM, Guntuku GS, Pothineni RB. New atherogenic indices: Assessment of cardio vascular risk in post menopausal dyslipidemia. Asian J Med Sci. 2015;6(6):25-32.

34. Stensvold I, Tverdal A, Urdal P, Graff-Iversen S. Non-fasting serum triglyceride concentration and mortality from coronary heart disease and any cause in middle aged Norwegian women. Br Med J. 1993;307(6915):1318-22. https://doi.org/ 10.1136/bmj.307.6915.1318.

35. Saklayen MG. The global epidemic of the metabolic syndrome. Curr Hypertens Rep. 2018;20(2):12. https://doi.org/ 10.1007/s11906-018-0812-z.

36. Yarahmadi S, Etemad K, Hazaveh AM, Azhang N. Urbanization and non-communicable risk factors in the capital city of 6 big provinces of Iran. Iran J Public Health. 2013;42(Supple1):113.

37. Kelishadi R, Alikhani S, Delavari A, Alaedini F, Safaie A, Hojatzadeh E. Obesity and associated lifestyle behaviours in Iran: findings from the first national non-communicable disease risk factor surveillance survey. Public Health Nutr. 2008;11(3):246-51. https://doi.org/ $10.1017 /$ S1368980007000262.

38. Neeland IJ, et al. Dysfunctional adiposity and the risk of prediabetes and type 2 diabetes in obese adults. JAMA. 2012;308(11):1150-9. https://doi.org/ 10.1001/2012.jama.11132.

39. Bouchi $R$, et al. Indirect measure of visceral adiposity 'A Body Shape Index'(ABSI) is associated with arterial stiffness in patients with type 2 diabetes. BMJ Open Diabetes Res Care 2016;4(1). https://doi.org/ 10.1136/bmjdrc-2015-000188.

40. Krakauer NY, Krakauer JC. A new body shape index predicts mortality hazard independently of body mass index. PloS one. 2012;7(7):e39504. https://doi.org/ 10.1371/journal.pone.0039504.

\section{Figures}




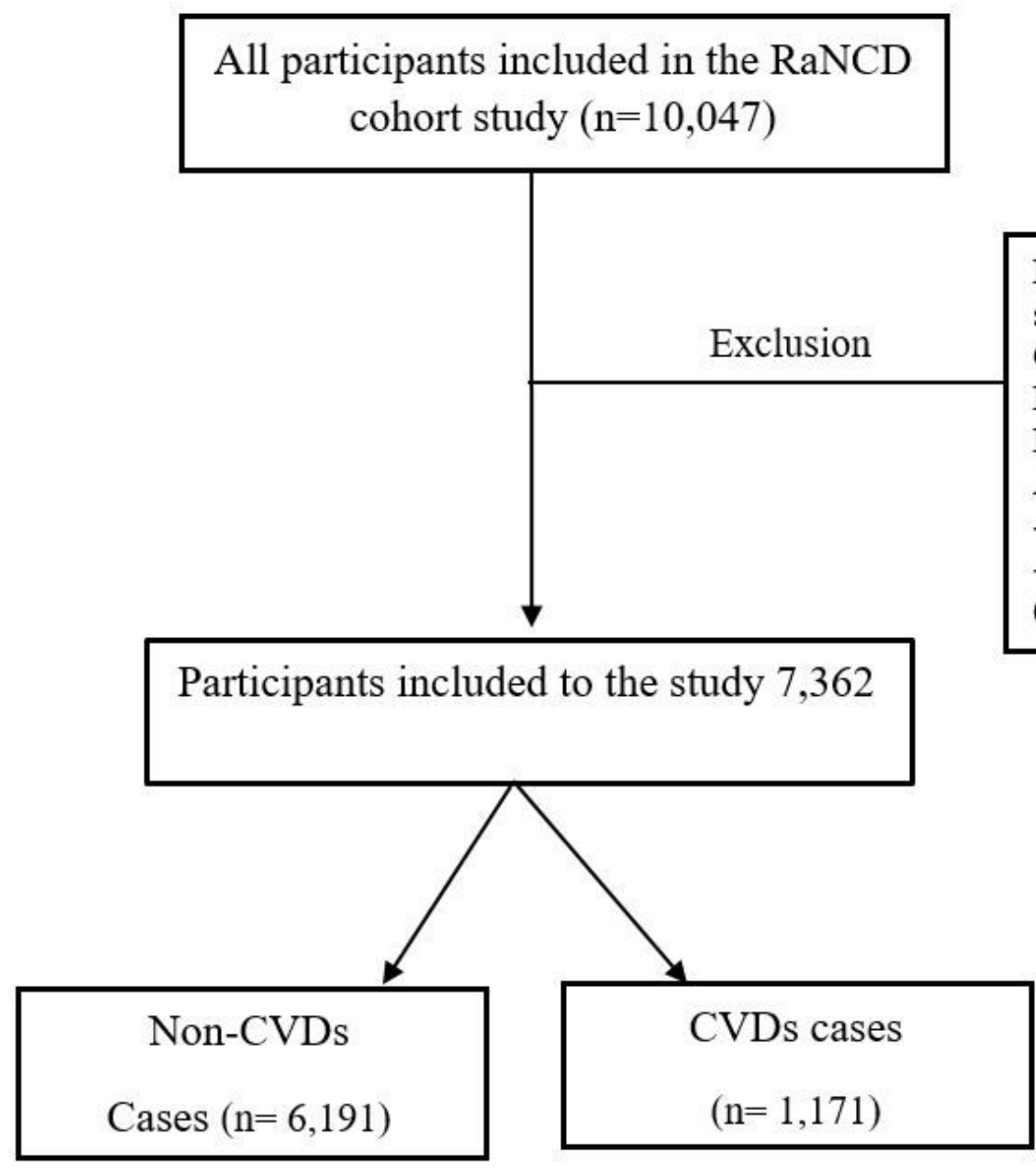

Patients were excluded from the study:

Cancer $(\mathrm{n}=85)$

Renal failure $(n=64)$

Kidney stones $(\mathrm{n}=1794)$

And:

- Pregnant women $(n=138)$

- Cases with missed information $(\mathrm{n}=557)$

\section{Figure 1}

Flow chart of study
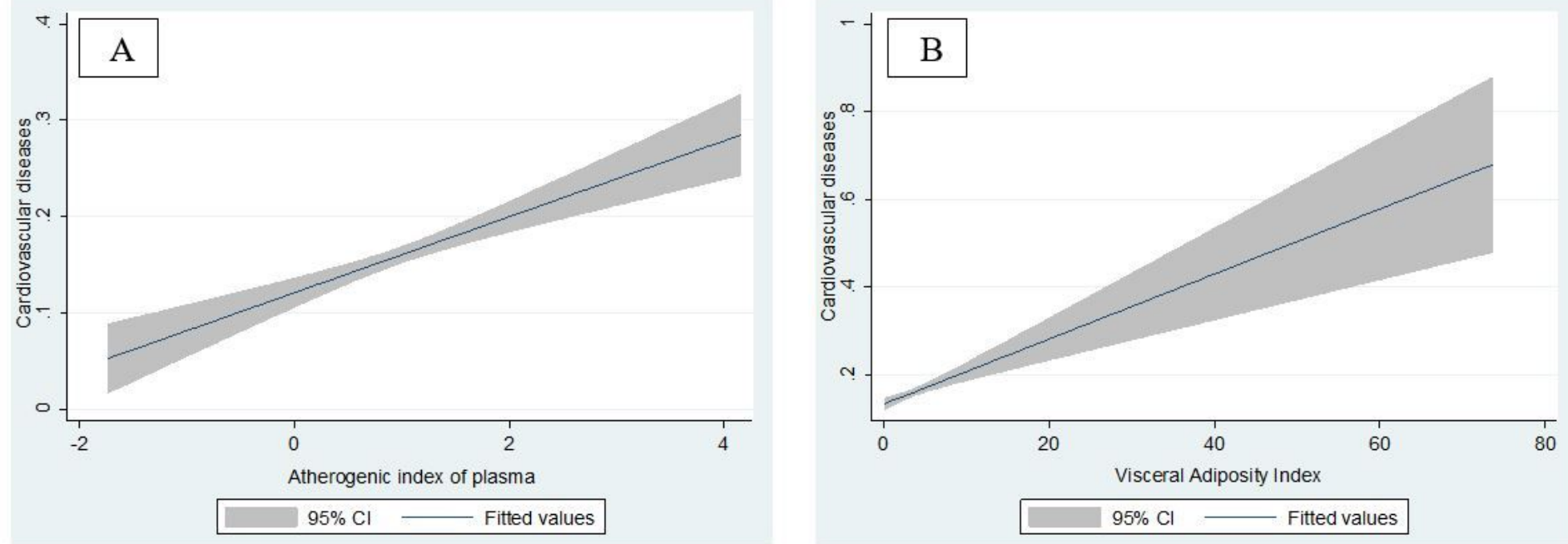

Figure 2 
Associations atherogenic index of plasma (A) and visceral adiposity index (B) with cardiovascular diseases 